\title{
How to choose kinematic or mechanical alignment individually according to preoperative characteristics of patients?
}

Chong Luan

Zibo Central Hospital

Dong-Tan Xu

Zibo Central Hospital

Ning-Jie Chen

Zibo Central Hospital

Fei-Fei Wang

Zibo Central Hospital

Kang-Song Tian

Zibo Central Hospital

Chao Wei

Zibo Central Hospital

Xian-Bin Wang ( $\sigma$ Wangxianbin@yandex.com )

Zibo Central Hospital

Research article

Keywords: Total knee arthroplasty, kinematic alignment, mechanical alignment, knee society score

Posted Date: February 21st, 2020

DOI: https://doi.org/10.21203/rs.2.24192/v1

License: (9) This work is licensed under a Creative Commons Attribution 4.0 International License.

Read Full License

Version of Record: A version of this preprint was published at BMC Musculoskeletal Disorders on July 7th, 2020. See the published version at https://doi.org/10.1186/s12891-020-03472-2. 


\section{Abstract}

Background Making decision in alignment techniques in total knee arthroplasty (TKA) remains controversial. This study aims to identify the potential patients who were suitable for the kinematic (KA) or mechanical alignment (MA).

Methods We reviewed 296 patients (296 TKAs, including 114 KA-TKAs and 182 MA-TKAs) who underwent unilateral TKA using a portable navigation system from 2016 to 2018 in our prospectively maintained database. The minimum followup was 1 year. Clinical outcomes including range of motion (ROM) and knee society score (KSS) were compared between KA-TKAs and MA-TKAs. Multiple regression models were used to evaluate the relationship between alignment techniques and KSS at the 1-year followup. Interaction and stratified analyses were conducted according to gender, age, body mass index (BMI), preoperative hip-knee-ankle (HKA) angle, ROM and KSS.

Results ROM and KSS at the 1-year followup didn't differ between MA-TKAs and KA-TKAs (all p>0.05). Alignment techniques did not associate with postoperative ROM (Adjusted $\beta=0.4,95 \%$ confidence interval [CI]: $-0.3,1.6 ; p=0.752$ ) or 1 -year KSS (Adjusted $\beta=2.2,95 \% \mathrm{Cl}:-0.7,5.6 ; p=0.107$ ). Patients with BMI more than $30 \mathrm{~kg} / \mathrm{m}^{\wedge} 2$ achieved better 1-year KSS when using MA than KA ( $p$ for interaction<0.05), Additionally, patients with preoperative HKA angle more than 10 degrees varus benefited more from KA than MA ( $p$ for interaction<0.05).

Conclusions Patients with severe varus deformity may be suitable for the KA technique, whereas MA should be used in obese patients.

\section{Introduction}

Total knee arthroplasty (TKA) is considered to be one of the most successful orthopedic surgery for pain relief and functional recovery in patients with knee arthritis. However, surgeons' perceptions of the success of the surgery is discordant with those of patients. Recently, many surgeons pay more attentions on the patient-reported outcome measures (PROMs) to evaluate results of the procedure [1]. According to the PROMs, it has reported that about $20 \%$ of patients with TKA were dissatisfied with the clinical outcomes of TKA $[2,3]$.

One possible explanation for these dissatisfied patients is that the contemporary TKA techniques fail to restore the nature knee kinematics [4]. Thus, there is an increasing debate regarding optimal alignment in TKA. Mechanical alignment (MA), the classical method proposed by Insall et al [5], aims to create a neutral hip-knee-ankle (HKA) axis. Kinematic alignment (KA) in TKA is an alternative technique to MA, which attempts to maintain the natural kinematic axis and ligament balance of the individual knee. Some studies indicated that the KA technique achieved greater range of motion (ROM) and higher rate of postoperative satisfaction compared with MA in TKA [6, 7]. However, several researches suggested similar results with these two alignment techniques [8]. Hence, we speculate that the KA or MA alignment 
technique may be not suitable for every case, which means surgeons should choose KA or MA individually.

To our best knowledge, there has been a lack of study on indications for the alignment parameter. Therefore, we conduct this study to identify the potential patients who were suitable for KA or MA technique in TKA. Additionally, we aimed to compare clinical outcomes in KA-TKA or MA-TKA using a multiple regression analysis with an adjustment for potential confounders.

\section{Methods}

\section{Patients}

After the Institutional Review Board approval, we reviewed 367 patients who underwent unilateral TKA using a portable navigation system from 2016 to 2018 in our prospectively maintained institutional navigation TKA database. All procedures were performed by three senior surgeons with extensive experience in navigation assisted TKA, including one surgeon using KA techniques and two using MA techniques. We excluded patients with post-traumatic, septic or inflammatory arthritis of the knee, $\mathrm{BMI}>$ $40 \mathrm{~kg} / \mathrm{m}^{\wedge} 2$, patients with valgus knee, patients with contralateral TKA or ipsilateral THA, those without a minimum followup of 1 year. After the aforementioned exclusion, 296 patients (296 TKAs, including 114 KA-TKAs and 182 MA-TKAs) were included in the final analysis.

\section{Surgical techniques}

All procedures were performed using the PS Vanguard TKA (Zimmer Biomet, Warsaw, Indiana). The medial parapatellar approach was conducted after placing tracker pins. An image-free navigation system (Zimmer Biomet, Warsaw, Indiana) was used to achieve coronal plane alignment according to surgeons' preference. In the MA-TKA, a neutral HKA axis with perpendicular components to the femoral and tibial mechanical axes was created. Femoral external rotation was set at $3^{\circ}$ to the femoral posterior condyles. With regarding to the KA-TKA, a restricted KA protocol described by Hutt et al. [9] was conducted in our institution. The HKA angles were limited for the KA-TKA from $6^{\circ}$ varus to $3^{\circ}$ valgus. We did not routinely resurface patellar in our institution. The postoperative care, including antibiotic administration, anticoagulation and physiotherapy, were based on an institutional protocol in all patients.

Radiographic evaluations using standing full-leg radiographs were performed and evaluated by 2 trained orthopedic fellows preoperatively and at the 1-year followup to determine the HKA angle. The Interobserver agreements were greater than 0.80 , and the mean of the 2 measurements was used for following analysis. Other medical records were reviewed manually to extract pertinent variables.

The main outcome was Knee Society Score (KSS) 2011 [10], including symptom (0-25), satisfaction (040), expectation (3-15) and functional activities (0-100) at the 1-year followup. Secondary outcomes included the range of motion (ROM) and HKA angle.

\section{Statistical analysis}


Statistical analysis was performed by using the statistical software packages R (http://www.Rproject.org). Student t-test or Mann-Whitney test was used to compare continuous variables, and the Fisher's exact test for categorical variables. The multiple regression analyses were conducted to evaluate the independent relationship between alignment methods and outcomes. The interaction and stratified analyses were used to identify patients who were suitable for KA or MA technique in TKA. A twopiecewise linear regression model was conducted and adjusted smoothing spline plots were created to graphically depict the associations between continuous variables and KSS scores at 1-year followup. A pvalue of 0.05 was considered significant.

\section{Results}

The patient characteristics was presented in Table 1. There was no significant difference in age, BMI, gender or ASA score among patients with KA-TKA or MA-TKA. Patients in the KA-TKA group had greater degrees of varus deformity compared with those in MA-TKA group ( $9.6 \pm 8.1$ varus vs. $7.2 \pm 5.3$ varus, $p=$ 0.046). Preoperative ROM and KSS score were comparable between groups.

Table 1

Patient demographics

\begin{tabular}{|llll|}
\hline & $\begin{array}{l}\text { KA-TKA } \\
\text { Group }(\mathbf{n}=114)\end{array}$ & $\begin{array}{l}\text { MA-TKA } \\
\text { Group }(\mathbf{n}=182)\end{array}$ & P-value \\
\hline Age (year) & $65.65 \pm 13.4$ & $64.92 \pm 14.73$ & 0.419 \\
\hline BMI $\left(\mathrm{kg} / \mathrm{m}^{2}\right)$ & $28.18 \pm 2.61$ & $28.06 \pm 3.36$ & 0.126 \\
\hline Female $(\mathrm{n}, \%)$ & $86(75.4 \%)$ & $129(70.9 \%)$ & 0.069 \\
\hline ASA score $\geq 3(\mathrm{n}, \%)$ & $13(11.4 \%)$ & $23(12.6 \%)$ & 0.527 \\
\hline Preoperative & & & \\
\hline ROM $\left({ }^{\circ}\right)$ & $109 \pm 19.1$ & $112 \pm 20.7$ & 0.963 \\
\hline HKA angle $\left({ }^{\circ}\right)$ & $9.6 \pm 8.1$ varus & $7.2 \pm 5.3$ varus & 0.046 \\
\hline KSS symptom $(25)$ & $9.1 \pm 3.8$ & $8.9 \pm 4.2$ & 0.857 \\
\hline KSS satisfaction $(40)$ & $15.6 \pm 7.2$ & $14.9 \pm 5.2$ & 0.461 \\
\hline KSS expectation $(15)$ & $12.8 \pm 1.7$ & $13.1 \pm 2.2$ & 0.581 \\
\hline KSS functional activities $(100)$ & $38.3 \pm 15.7$ & $41.6 \pm 14.3$ & 0.265 \\
\hline Total KSS score (180) & $73.2 \pm 30.4$ & $79.5 \pm 28.6$ & 0.624 \\
\hline
\end{tabular}


Table 2

Clinical outcomes among patients with KA or MA at the 1-year followup

\begin{tabular}{|lllll|}
\hline & $\begin{array}{l}\text { KA-TKA } \\
\text { Group }(\boldsymbol{n}= \\
\text { 114) }\end{array}$ & $\begin{array}{l}\text { MA-TKA } \\
\text { Group }(\boldsymbol{n}= \\
\text { 182) }\end{array}$ & $\begin{array}{l}\text { *Adjusted } \boldsymbol{\beta}(95 \% \\
\text { Cl) }\end{array}$ & P-value \\
\hline ROM $\left(^{\circ}\right)$ & $125.6 \pm 19.1$ & $124.9 \pm 17.7$ & $0.4(-0.3,1.6)$ & 0.752 \\
\hline HKA angle $\left(^{\circ}\right)$ & $1.2 \pm 2.5$ varus & $0.3 \pm 1.9$ varus & $1.5(0.3,1.7)$ & 0.027 \\
\hline KSS symptom (25) & $18.1 \pm 4.5$ & $18.7 \pm 4.7$ & $-0.6(-1.3,0.5)$ & 0.916 \\
\hline KSS satisfaction (40) & $25.6 \pm 6.1$ & $25.9 \pm 6.7$ & $-0.2(-0.6,1.2)$ & 0.639 \\
\hline KSS expectation (15) & $10.2 \pm 2.6$ & $9.9 \pm 1.8$ & $0.5(-0.8,2.1)$ & 0.092 \\
\hline $\begin{array}{l}\text { KSS functional activities } \\
(100)\end{array}$ & $66.4 \pm 6.9$ & $65.7 \pm 7.1$ & $1.1(-0.9,2.7)$ & 0.374 \\
\hline Total KSS score (180) & $126.8 \pm 16.4$ & $124.3 \pm 14.3$ & $2.2(-0.7,5.6)$ & 0.107 \\
\hline
\end{tabular}

Clinical outcomes at the 1-year followup didn't differ between groups (Table 2). The postoperative ROM of KA and MA was $(125.6 \pm 19.1)$ degrees and $(124.9 \pm 17.7)$ degrees, respectively. The total KSS of KA and MA was $(126.8 \pm 16.4)$ degrees and $(124.3 \pm 14.3)$ degrees, respectively. After adjusting for confounding variables in Table 1, the clinical outcomes showed no significant difference in ROM (Adjusted $\beta=0.4,95 \% \mathrm{Cl}:-0.3,1.6 ; p=0.752$ ) or total KSS (Adjusted $\beta=2.2,95 \% \mathrm{Cl}:-0.7,5.6 ; p=0.107$ ) between groups. Due to differing targets for alignment, the postoperative HKA angle of KA-TKA and MATKA was significant different ( $1.2 \pm 2.5$ vs. $0.3 \pm 1.9, p=0.027)$.

The stratified and Interaction analyses suggested that the association between alignment techniques (MA as reference) and total KSS score was modified by obesity and preoperative HKA angle (Table 3). Patients with $B M I \geq 30 \mathrm{~kg} / \mathrm{m}^{2}$ had a lower $\beta$ between the KA-TKA and KSS than those with $B M I<30 \mathrm{~kg} / \mathrm{m}$ ( $\beta$ : -1.66 vs. $1.48, p$ for interaction $=0.028$ ). Additionally, the $\beta$ between the KA-TKA and KSS was higher in patients with preoperative $H K A \geq 10$ degrees varus than those with $H K A<10$ degrees varus ( $\beta: 2.44$ vs. 0.52 , $\mathrm{p}$ for interaction $=0.013$ ). The adjusted smoothing splines were revealed relationship between BMI (Fig. 1 and Fig. 2) or preoperative HKA angle (Fig. 3 and Fig. 4) and total KSS stratified by alignment techniques. Figure 1 showed a non-linear relationship between BMI and KSS in patients with KA-TKA. When BMI was greater than the turning point at BMI of $32.5 \mathrm{~kg} / \mathrm{m}^{\wedge} 2$, the $\mathrm{KSS}$ decreased significantly with the increase in BMI (Fig. 1). Additionally, there were nonlinear relationships between preoperative HKA angle and 1-year KSS. (Fig. 2) The two-piecewise linear regression model didn't find the turning point. 
Table 3

Interaction and stratified analyses between alignment techniques (MA as reference) and KSS score

\begin{tabular}{|llll|}
\hline Subgroup & $\boldsymbol{\beta}, 95 \% \mathrm{Cl}$ & P-value & P-value for interaction \\
\hline Sex & & & 0.684 \\
\hline Male & $1.01(-1.06,2.08)$ & 0.793 & \\
\hline Female & $1.48(-4.89,6.92)$ & 0.813 & \\
\hline Age, years & & & 0.076 \\
\hline$<65$ & $1.53(-2.40,5.45)$ & 0.793 & \\
\hline$\geq 65$ & $1.07(-1.30,2.76)$ & 0.452 & \\
\hline BMI, kg/m^2 & & & 0.028 \\
\hline$<30$ & $1.48(-1.81,5.17)$ & 0.274 & \\
\hline$\geq 30$ & $-1.66(-4.08,-0.46)$ & 0.039 & \\
\hline HKA angle & & & 0.013 \\
\hline$<10^{\circ}$ varus & $0.52(-0.99,0.95)$ & 0.983 & \\
\hline$\geq 10^{\circ}$ varus & $2.44(0.53,4.63)$ & 0.042 & \\
\hline Preoperative ROM & & & 0.668 \\
\hline$<90^{\circ}$ & $0.82(-1.74,2.59)$ & 0.702 & \\
\hline$\geq 90^{\circ}$ & $0.42(-2.48,3.31)$ & 0.78 & \\
\hline Preoperative total KSS & & & 0.583 \\
\hline Tertile low & $-0.84(-2.04,-0.15)$ & 0.328 & \\
\hline Tertile middle & $1.04(-0.12,3.21)$ & 0.565 & \\
\hline Tertile high & $0.21(-2.13,2.55)$ & 0.859 & \\
\hline & & & \\
\hline
\end{tabular}

\section{Discussion}

Recently, there has been a raising debate on MA or KA techniques. The data in the literature was inconsistent, which makes surgeons confused to make decisions. This may be explained that we should choose alignment techniques individually. The most important finding of the present study was that patients with preoperative varus deformity more than 10 degrees were more suitable for KA-TKA, while patients with BMI more than $30 \mathrm{~kg} / \mathrm{m}^{\wedge} 2$ would benefit more from MA-TKA. 
There have been numerous studies on the comparison between the MA-TKA and the KA-TKA. Several studies have suggested a substantial portion of the normal population didn't have a neutral mechanical alignment. Bellemans et al. reported $32 \%$ of men and $17 \%$ of women had varus knees with a natural mechanical axis of $3^{\circ}$ varus or more [11]. Nam et al. suggested only $31 \%$ of knees had both a neutral mechanical alignment and the absence of joint line obliquity [12]. Thus, several scholars hold the view that KA-TKA may restore normal knee kinematics. Faschingbauer et al. indicated KA-TKA could achieve similar kinematics of the patellofemoral joint relative to the normal state [13]. Blakeney et al. suggested KA-TKA reproduced more closely normal gait of healthy controls compared to MA-TKA due to the restoration of the individual's knee kinematics and ligament tension in KA-TKA [14]. Ishikawa et al. revealed greater femoral rollback and more external rotation of the femoral component in KA-TKA than Ma-KTA by using a musculoskeletal computer simulation [15].

Although the aforementioned studies provided evidences in favor of KA-TKA, the present study found the clinical outcomes were comparable between the two groups which was similar with data in the recent literature. Luo et al. conducted a meta-analysis including 9 randomized controlled trials with $1170 \mathrm{KA}-$ TKAs and 1171 MA-TKAs. This meta-analysis suggested the KSS, knee injury and osteoarthritis outcome score (KOOS), EuroQoL 5-dimension questionnaire (ED-5D), ROM, and complications were similar for KATKA and MA-TKA. In a recent study by McEwen et al. [16], they prospectively enrolled 41 patients who were scheduled to undergo simultaneous TKAs. They randomized one side using MA and the other side using KA. With a minimum of 2-year followup, although more patients preferred their KA knees, they suggested no difference in ROM or functional scores between groups. Additionally, there is a lack of data on comparisons the long-term clinical outcomes. Ishikawa et al. [15] suggested KA-TKA increased patellofemoral and tibiofemoral contact stresses by using finite element analysis, which may impair longterm outcomes. Berend et al. reviewed 3152 TKAs for osteoarthritis with a mean 5-year followup and indicated that varus tibial component alignment more than 3.0 degrees had a 17 -fold risk of subsequent tibial component aseptic loosening [17]. Another study with the mean followup of 7.6 years found failure was least likely to occur in patients with a neutral alignment of both the tibial and the femoral component [18].

Several studies have reported the survival rate of TKA was lower in obese patients than nonobese patients [19-21]. However, the interaction between obesity and alignment on the survival of TKA remains unknown. The present study revealed that obese patients may be more suitable for MA-TKA than KA-TKA. Interestingly, our results were similar to a previous study by Berend et al [17]. They found BMI alone was not associated with failure, but BMI more than $33.7 \mathrm{~kg} / \mathrm{m}^{\wedge} 2$ combined with varus tibial component more than 3 degrees had a 168-fold risk of subsequent failure. The possible reason may be that overloading of the knee occurs in patients with high BMI and varus axis, resulting in greater impact loading across the tibial component, therefore, caused patients' discomfort and might increase component loosening and lower implant survival rate. Additionally, we found patients with preoperative HKA angle more than 10 degrees varus may benefit more from KA-TKA than MA-TKA. The reason remains unclear. It may be explained that patients with severe deformity frequently possessed critical contracture of knees. Thus, these patients need more soft tissue releases that may impair patients' satisfaction. 
Several limitations should be noted. First, this is a single-institution study and thus its findings need external validation. Second, only single implant manufacturer was used in the present study, which may limit the generalizability of the findings. Third, this is not a randomized study, and there may be selection bias.

\section{Conclusions}

In conclusion, KA-TKA may be not suitable for obese patients, whereas patients with severe varus deformity may benefit more from KA-TKA. These findings need to be borne in mind when deciding which alignment techniques should be used in TKA. Further studies with the long-term followup are required to validate our results.

\section{Declarations}

\section{Acknowledgments}

The authors would like to thank all staff from the participating departments and clinics.

\section{Funding sources}

Not applicable.

\section{Availability of data and materials}

We do not wish to share our data, because some of the patient's data regarding individual privacy, and according to the policy of our hospital, the data could not be shared with others without permission.

\section{Authors' contributions}

CL drafted the manuscript. CL, DTX, NJC, FFW, KST and CW performed data collection and data analysis. CL, DTX, NJC, FFW, KST and XBW conceived of the study, participated in the design of the study, performed data interpretation, and participated in coordination. All authors read and approved the final manuscript.

\section{Ethics approval and consent to participate}


This study was approved by the Ethics Committee of the ZIBO central hospital and in accordance with the standards of the National Research Council. Written informed consent was obtained from all participants.

\section{Consent for publication}

Not applicable.

\section{Competing interests}

The authors declare that they have no competing interests

\section{References}

1. Kahlenberg CA, Nwachukwu BU, McLawhorn AS, Cross MB, Cornell CN, Padgett DE. Patient Satisfaction After Total Knee Replacement: A Systematic Review. HSS J. 2018;14:192-201.

2. Bourne RB, Chesworth BM, Davis AM, Mahomed NN, Charron KDJ. Patient satisfaction after total knee arthroplasty: who is satisfied and who is not? Clin Orthop Relat Res. 2010;468:57-63.

3. Baker PN, van der Meulen JH, Lewsey J, Gregg PJ, National Joint Registry for England and Wales. The role of pain and function in determining patient satisfaction after total knee replacement. Data from the National Joint Registry for England and Wales. J Bone Joint Surg Br. 2007;89:893-900.

4. Hirschmann MT, Testa E, Amsler F, Friederich NF. The unhappy total knee arthroplasty (TKA) patient: higher WOMAC and lower KSS in depressed patients prior and after TKA. Knee Surg Sports Traumatol Arthrosc. 2013;21:2405-11.

5. Insall J, Scott WN, Ranawat CS. The total condylar knee prosthesis. A report of two hundred and twenty cases. J Bone Joint Surg Am. 1979;61:173-80.

6. Almaawi AM, Hutt JRB, Masse V, Lavigne M, Vendittoli P-A. The Impact of Mechanical and Restricted Kinematic Alignment on Knee Anatomy in Total Knee Arthroplasty. J Arthroplasty. 2017;32:2133-40.

7. Howell SM, Hodapp EE, Vernace JV, Hull ML, Meade TD. Are undesirable contact kinematics minimized after kinematically aligned total knee arthroplasty? An intersurgeon analysis of consecutive patients. Knee Surg Sports Traumatol Arthrosc. 2013;21:2281-7.

8. Luo Z, Zhou K, Peng L, Shang Q, Pei F, Zhou Z. Similar results with kinematic and mechanical alignment applied in total knee arthroplasty. Knee Surg Sports Traumatol Arthrosc. 2019;

9. Hutt JRB, LeBlanc M-A, Massé V, Lavigne M, Vendittoli P-A. Kinematic TKA using navigation: Surgical technique and initial results. Orthop Traumatol Surg Res. 2016;102:99-104.

10. Noble PC, Scuderi GR, Brekke AC, Sikorskii A, Benjamin JB, Lonner JH, et al. Development of a new Knee Society scoring system. Clin Orthop Relat Res. 2012;470:20-32. 
11. Bellemans J, Colyn W, Vandenneucker H, Victor J. The Chitranjan Ranawat award: is neutral mechanical alignment normal for all patients? The concept of constitutional varus. Clin Orthop Relat Res. 2012;470:45-53.

12. Nam D, Shah RR, Nunley RM, Barrack RL. Evaluation of the 3-dimensional, weight-bearing orientation of the normal adult knee. J Arthroplasty. 2014;29:906-11.

13. Faschingbauer M, Hacker S, Seitz A, Dürselen L, Boettner F, Reichel $H$. The tibial cut influences the patellofemoral knee kinematics and pressure distribution in total knee arthroplasty with constitutional varus alignment. Knee Surg Sports Traumatol Arthrosc. 2019;

14. Blakeney W, Clément J, Desmeules F, Hagemeister N, Rivière C, Vendittoli P-A. Kinematic alignment in total knee arthroplasty better reproduces normal gait than mechanical alignment. Knee Surg Sports Traumatol Arthrosc. 2019;27:1410-7.

15. Ishikawa M, Kuriyama S, Ito H, Furu M, Nakamura S, Matsuda S. Kinematic alignment produces nearnormal knee motion but increases contact stress after total knee arthroplasty: A case study on a single implant design. Knee. 2015;22:206-12.

16. McEwen PJ, Dlaska CE, Jovanovic IA, Doma K, Brandon BJ. Computer-Assisted Kinematic and Mechanical Axis Total Knee Arthroplasty: A Prospective Randomized Controlled Trial of Bilateral Simultaneous Surgery. J Arthroplasty. 2019;

17. Berend ME, Ritter MA, Meding JB, Faris PM, Keating EM, Redelman R, et al. Tibial component failure mechanisms in total knee arthroplasty. Clin Orthop Relat Res. 2004;26-34.

18. Ritter MA, Davis KE, Meding JB, Pierson JL, Berend ME, Malinzak RA. The effect of alignment and BMI on failure of total knee replacement. J Bone Joint Surg Am. 2011;93:1588-96.

19. Boyce L, Prasad A, Barrett M, Dawson-Bowling S, Millington S, Hanna SA, et al. The outcomes of total knee arthroplasty in morbidly obese patients: a systematic review of the literature. Arch Orthop Trauma Surg. 2019;139:553-60.

20. Gøttsche D, Gromov K, Viborg PH, Bräuner EV, Pedersen AB, Troelsen A. Weight affects survival of primary total knee arthroplasty: study based on the Danish Knee Arthroplasty Register with 67,810 patients and a median follow-up time of 5 years. Acta Orthop. 2019;90:60-6.

21. Si H, Zeng Y, Shen B, Yang J, Zhou Z, Kang P, et al. The influence of body mass index on the outcomes of primary total knee arthroplasty. Knee Surg Sports Traumatol Arthrosc. 2015;23:182432.

\section{Figures}




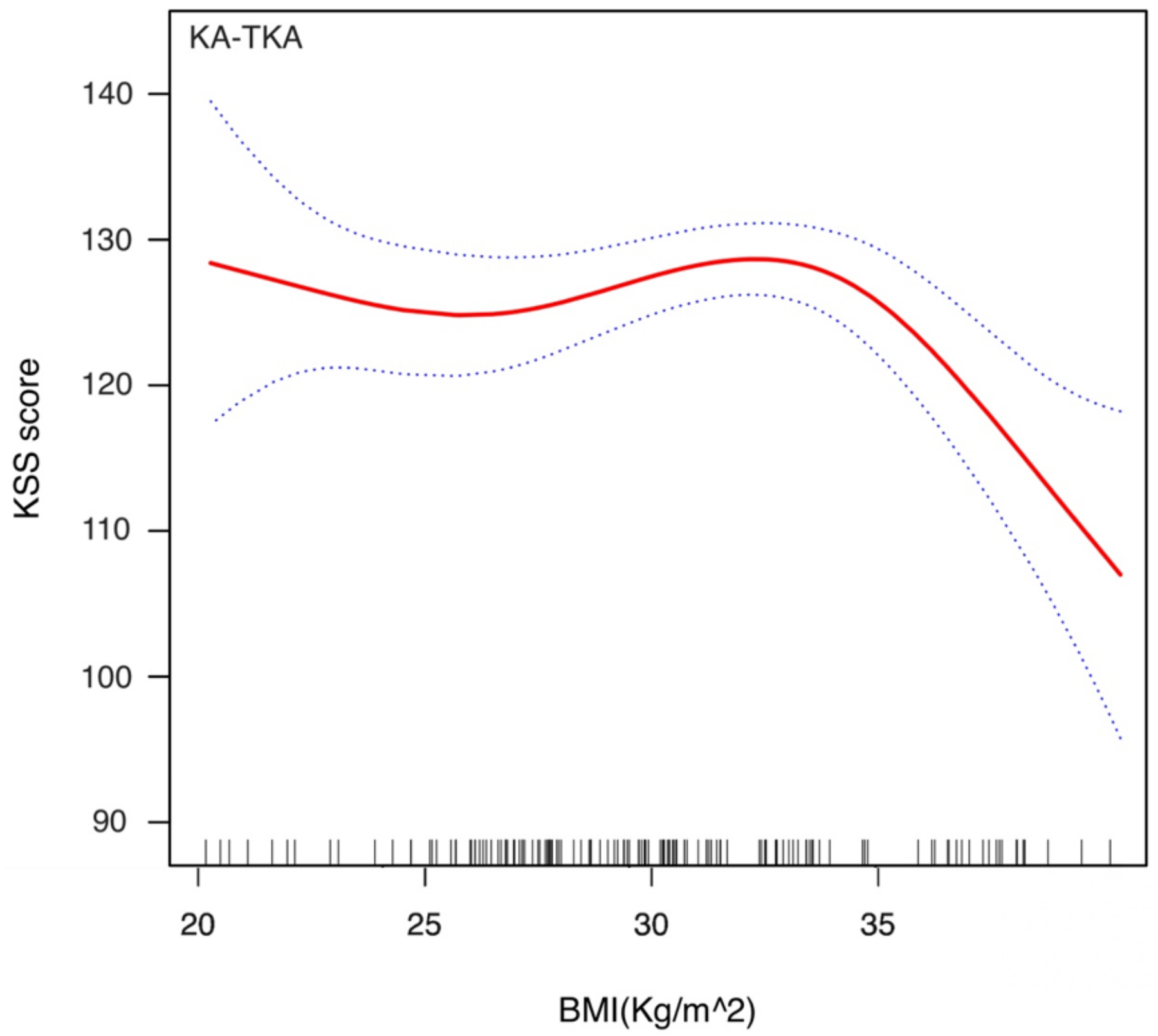

Figure 1

Adjusted smoothing spline between BMI and 1-year KSS in the KA-TKA group 


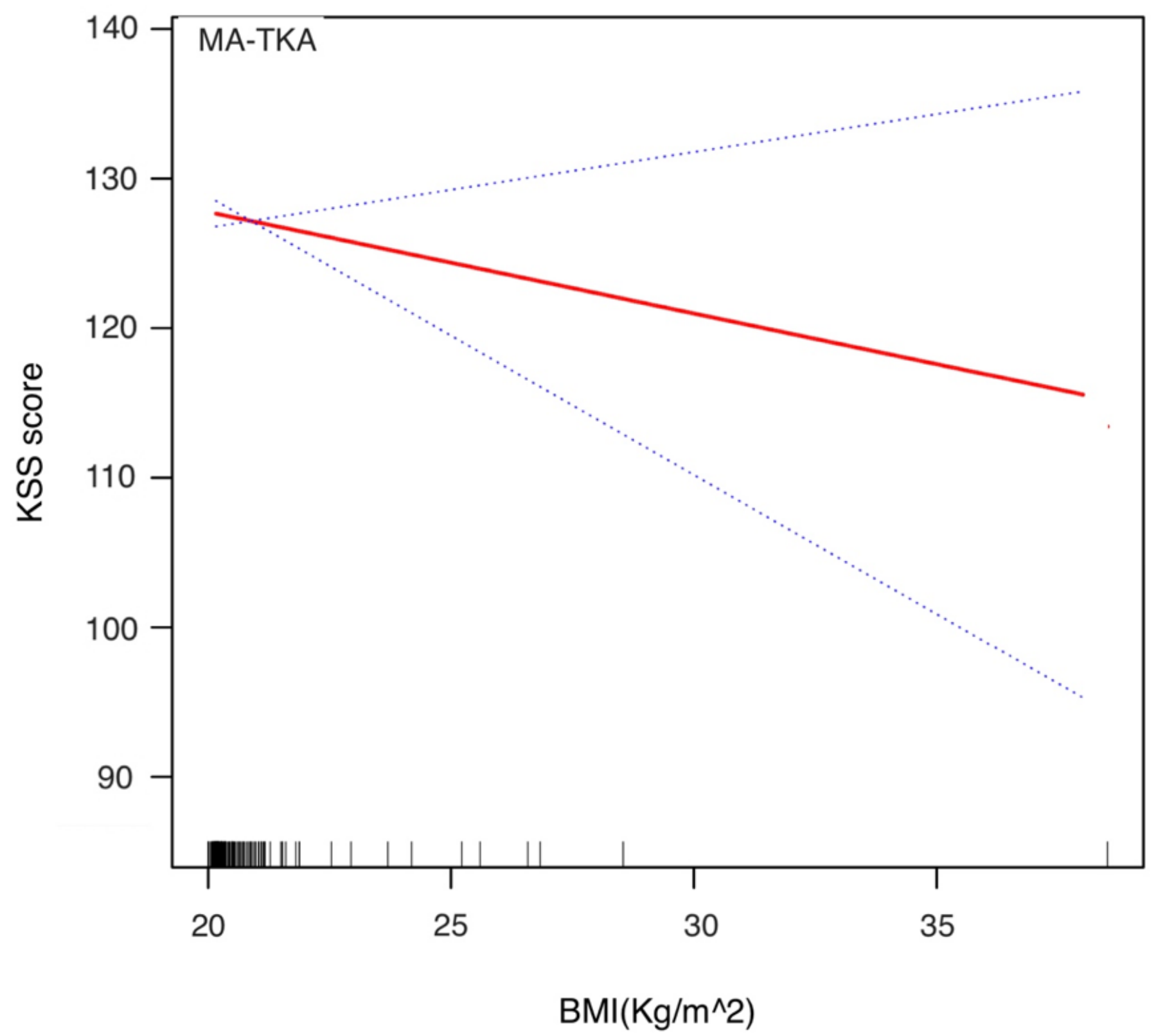

Figure 2

Adjusted smoothing spline between BMI and 1-year KSS in the MA-TKA group 


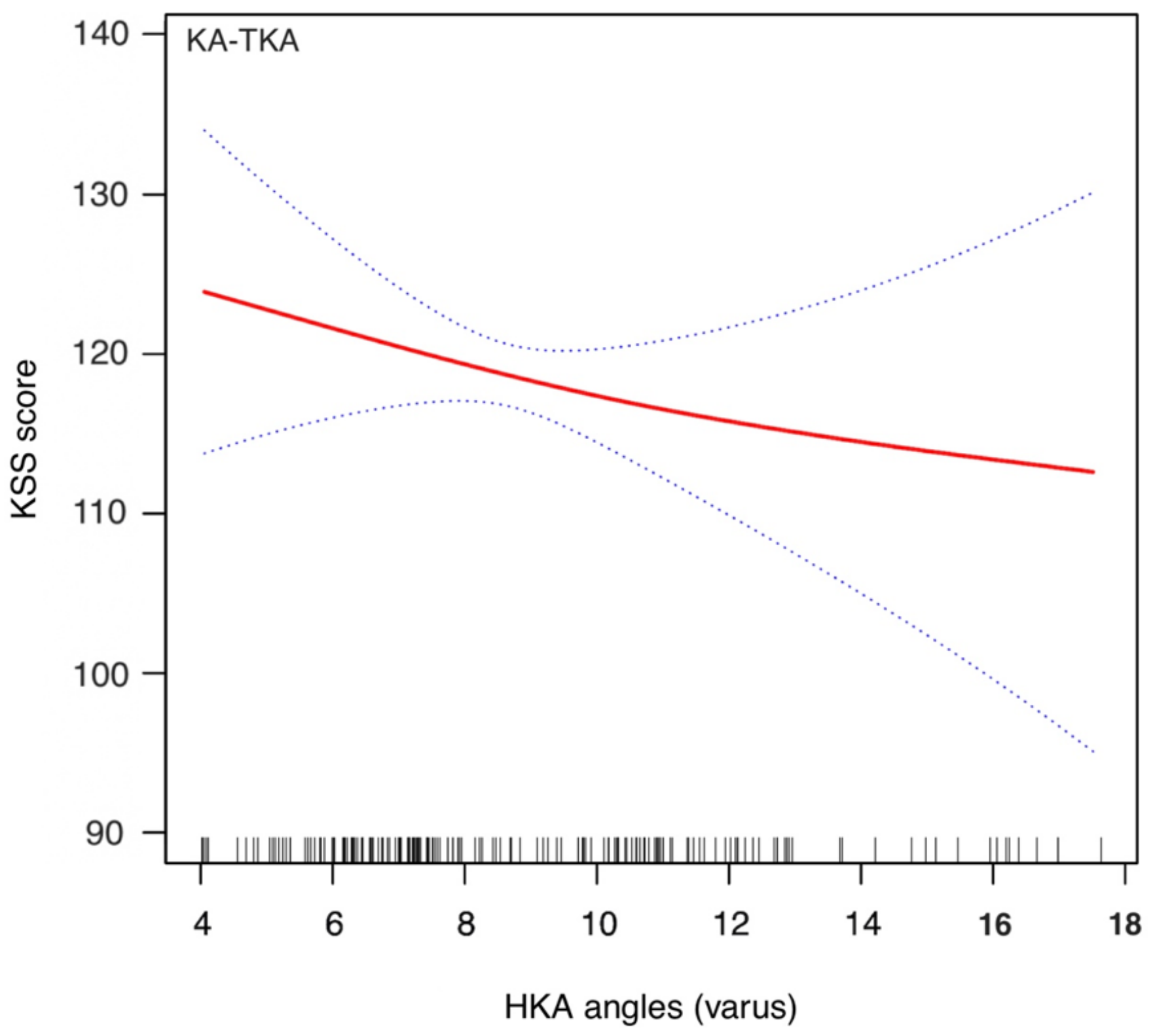

Figure 3

Adjusted smoothing spline between preoperative HKA angle (varus deformity) and 1-year KSS in the KATKA group 


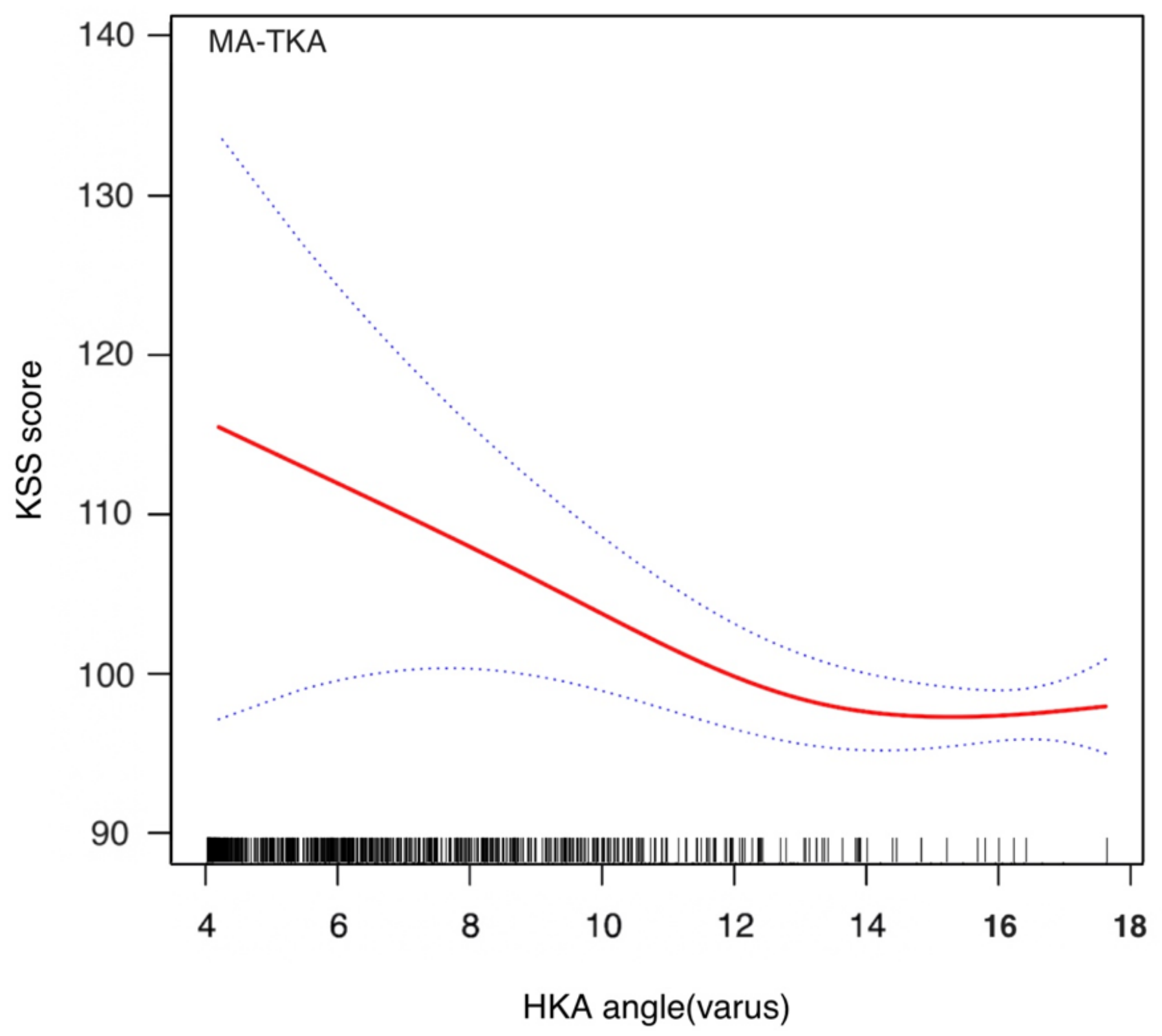

Figure 4

Adjusted smoothing spline between HKA preoperative angle (varus deformity) and 1-year KSS in the MATKA group 